\title{
Analysis of Temperature Rise in High-Speed Permanent Magnet Synchronous Traction Motors by Coupling the Equivalent Thermal Circuit Method and Computational Fluid Dynamics
}

\author{
Jungang Jia* \\ Shandong Polytechnic, Jinan, 250104, China \\ *Corresponding Author: Jungang Jia. Email: A17897555891@163.com \\ Received: 30 December 2019; Accepted: 06 August 2020
}

\begin{abstract}
To solve the problem of temperature rise caused by the high power density of high-speed permanent magnet synchronous traction motors, the temperature rise of various components in the motor is analyzed by coupling the equivalent thermal circuit method and computational fluid dynamics. Also, a cooling strategy is proposed to solve the problem of temperature rise, which is expected to prolong the service life of these devices. First, the theoretical bases of the approaches used to study heat transfer and fluid mechanics are discussed, then the fluid flow for the considered motor is analyzed, and the equivalent thermal circuit method is introduced for the calculation of the temperature rise. Finally, the stator, rotor loss, motor temperature rise, and the proposed cooling method are also explored through experiments. According to the results, the stator temperature at $50,000 \mathrm{r} / \mathrm{min}$ and $60,000 \mathrm{r} / \mathrm{min}$ at no-load operation is $68^{\circ} \mathrm{C}$ and $76^{\circ} \mathrm{C}$, respectively. By monitoring the temperature of the air outlets inside and outside the motor at different speeds, it is also found that the motor reaches a stable temperature rise after $65 \mathrm{~min}$ of operation. Coupling of the thermal circuit method and computational fluid dynamics is a strategy that can provide the average temperature rise of each component and can also comprehensively calculate the temperature of each local point. We conclude that a hybrid cooling strategy based on axial air cooling of the inner air duct of the motor and water cooling of the stator can meet the design requirements for the ventilation and cooling of this type of motors.
\end{abstract}

Keywords: Thermal circuit method; computational fluid dynamics; high-speed permanent magnet synchronous traction motor; rotor temperature rise; stator temperature rise

\section{Introduction}

In recent years, the industry of power and electronic devices has developed rapidly. Meanwhile, the research and development of high-speed motors have also received widespread attention. Compared with traditional ordinary motors, high-speed motors have smaller power losses and less noises, and can also be directly connected to high-speed loads, eliminating the need for complex variable-speed gearing devices

This work is licensed under a Creative Commons Attribution 4.0 International License, which permits unrestricted use, distribution, and reproduction in any medium, provided the original work is properly cited. 
in ordinary motors, which helps in improving the overall efficiency of the system in which the motor is located [1]. With the continuous improvement of the performance of permanent magnetic materials, the development of high-speed permanent magnet synchronous motors is growing rapidly. The advantages of permanent magnet synchronous motors are their simple structures and high power quality. They do not require field windings and direct circuit (DC) excitation power sources and can directly eliminate faultprone devices, such as slip rings and brushes [2]. At present, high-speed permanent magnet synchronous motors are vital in high-demand fields, such as aerospace, ships, and industrial and agricultural productions.

High-speed permanent magnet motors have the characteristics of small size and high power density. At the same time, the loss density also increases accordingly, which affects the temperature rise of the motor. Especially for a fully-enclosed permanent magnet traction motor, if the temperature rise is too high, the magnetic density of the permanent magnet will be reduced due to the influence of the high-temperature environment, which may even lead to irreversible demagnetization [3]. This does great harm to the performance of any system and seriously affects its safety and stability. When the temperature rise of the motor weakens the performance of the magnet, the stator winding insulation of the motor may be damaged, and the motor parameters may be changed, which will eventually cause the motor to run steadily. Therefore, the research on the temperature rise of the motor is of great significance to the permanent magnet synchronous traction motor. It is directly related to the performance of the motor and affects the life and reliability of the motor [4].

Computational fluid dynamics (CFD) is an independent discipline based on a computer server that applies classical fluid mechanics theory and numerical discrete methods to explore the laws of fluid motion [5]. CFD is applied to the analysis of the temperature rise in permanent magnet synchronous motors. Real physical quantities are simulated by a set of discrete and finite variables. Based on certain principles, a set of equations describing the relationship between field variables at discrete points is established. Thus, an approximate solution to the field variables is obtained [6].

In this study, a thermal circuit model of a high-speed synchronous permanent magnet step motor will be used to solve the transient and steady-state temperature rise of the motor during long-term operation, providing a reference for the temperature rise of the motor during overload operation. In addition, the high-speed permanent magnet traction motor is analyzed by CFD, and the temperatures at local points are comprehensively calculated, which provides a reference for the location of the thermocouple in the experiment. Finally, a zigzag ventilation duct is designed, and the optimal parameters are determined based on the method of CFD with the thermal circuit method, hoping to improve the thermal performance of the motor.

\section{Methods}

\subsection{Basics of Heat Transfer and Fluid Mechanics}

Heat transfer is a subject that investigates the laws that heat transfers; it has been widely used in chemical, power electronics, aerospace, and other fields [7]. French scholar Fourier proposed the method of separating variables for solving partial differential equations in 1807, and then successfully constructed the theory of heat conduction. According to heat transfer theory, heat is transferred from high-temperature objects to low-temperature objects through three ways, i.e., heat conduction, heat convection, and heat radiation [8]. Heat conduction refers to the phenomenon of energy transfer through the thermal movement of molecules, atoms, and free electrons inside the object when there is no relative displacement between various parts of the object. Heat convection refers to the heat transfer process of relative displacement of internal particles when the fluid flows. Heat radiation refers to the transfer of energy by an object through electromagnetic waves. Applied heat transfer can be achieved through experiments, analysis, and numerical calculations when studying practical problems. Among the various methods, the numerical method is based on computer application technology. It uses various discretization methods to establish a 
numerical model and finally obtains a set of numbers in time and space, thereby getting a numerical solution for the quantitative description of the flow field [9]. The study of fluid mechanics focuses on the basic laws of flowing objects. The basic idea of computational fluid mechanics: replace the original continuous physical quantity fields in the time domain and the space domain, such as the velocity field and the pressure field, with a series of finite discrete points of variable value sets. Through some principles and methods, the algebraic equations about the relations among the field variables at these discrete points are established, and the approximate solution of the field variables is obtained by solving the algebraic equations.

Since the motion of fluid has the characteristics of continuous deformation, it is more complicated to analyze the velocity, pressure, energy, and interaction between the fluid and solid during fluid movement. When solving the problems of fluid mechanics, it is necessary to follow the universal flow laws, including conservation of mass, conservation of energy, and laws of thermodynamics. If the fluid is in a turbulent state, the fluid mechanics problem needs to be solved by the equation of motion. If the flow contains a mixture or interaction of different components (components), the system must also observe the law of conservation of components. If the flow is in a turbulent state, the system must also follow the additional turbulent transport equation. The law of conservation of mass equations can be expressed as: the increase in mass in the fluid micro-units per unit time is equal to the net mass flowing into the microunits in the same time interval. The mass conservation equation (continuity equation), momentum conservation equation (motion equation), and energy conservation equation (energy equation) are respectively expressed as:

$\nabla \cdot v=0$

$(\nabla \cdot v) v=F-\frac{1}{\rho} \nabla p+\frac{\mu}{\rho} \nabla^{2} v$

$c_{p} \rho(\nabla \cdot v) T=\lambda \nabla^{2} T+S_{T}$

where: $\mu$ is the hydrodynamic viscosity, $\lambda$ is the thermal conductivity of the fluid, and $S_{T}$ is the viscous dissipation term. In electrical engineering, the fluid inside and outside the motor is mostly in a state of turbulent motion. Most of the turbulence models are built from the conservation of mass equation to the conservation of momentum equation. Based on reasonable assumptions, the turbulence model is suitable for a certain range. Among them, the Reynolds averaged Navier stokes (RANS) equations are the most widely used equations. The energy equation is independent of the mass equation and momentum equation. Under the proper definite solution conditions, after the correct velocity field and pressure field are solved, the temperature distribution can be obtained independently from the energy equation. Therefore, the focus is on the simultaneous solution of the continuity equation and the momentum equation. In fact, due to a strong coupling relation between the mass equation and the momentum equation, in addition to a nonlinear convection term in the momentum equation, it is very difficult to solve the equation directly. The common method is separation solution, whose basic idea is to have the pressure field and velocity field approach the true solution through an iterative method at the same time. From the perspective of simplified programming, each field control equation in the fluid field can be written a general transport equation of the form:

$\operatorname{div}(\rho v \phi)=\operatorname{div}(\Gamma \operatorname{grad} \phi)+S$

where: $\rho$ is fluid density, $v$ is fluid velocity vector, $\phi, \Gamma$, and $S$ are respectively generalized variable, generalized diffusion coefficient, and generalized source term.

According to the law of conservation of mass, by using the finite volume control analysis method, a continuity equation in integral form can be obtained [10], which is expressed as: 
$\frac{\partial}{\partial t} \int_{\tau} \rho d \tau+\oint_{A} \rho(\mathrm{V} \cdot n) d A=0$

where $\rho$ denotes the density of the control body $\tau$ in the flow field, $\mathrm{V}$ denotes the speed, and $A$ denotes the surface area of the control body. The continuity equation is irrelevant to the force; therefore, it is suitable for viscous fluid motion as well as fluid motion that ignores viscosity.

In conformity with the law of conservation of momentum, for a certain fluid, the time rate of the total momentum should be equal to the sum of the volume force and the surface force acting on it. The integral form of the following equation of motion is obtained by using the equation of the volume integral material derivative:

$\frac{\partial}{\partial t} \int_{r} \rho d \tau+\oint_{A} \rho(\mathrm{V} \cdot n) d A=\int_{r} \rho d \tau+\oint_{A} p_{n} d A$

In the process of studying fluid motion, thermal efficiency is also a problem to be considered. Based on satisfying the motion equation and the continuity equation, the energy equation should also be satisfied. It makes sure that the time change rate of the total energy of the fluid is equal to the sum of the work done by the external force and the heat transferred to the system per unit time, which can be expressed as:

$\frac{\partial}{\partial t} \int_{r}\left(e+\frac{v^{2}}{2}\right) d \tau+\oint_{A} \rho\left(e+\frac{v^{2}}{2}\right)(\mathrm{V} \cdot n) d A=\int_{r} \rho d \tau+\int_{A} k \nabla T \cdot n d A+\int_{r} \rho f \cdot V d \tau+\oint_{A} p_{n} \cdot V d A$

where $e$ represents the thermodynamic internal energy, $\frac{v^{2}}{2}$ represents the kinetic energy, $n$ is a unit vector in the direction of the outer normal of the surface $A, k$ represents the thermal conductivity of the fluid, $f$ and $p_{n}$ represent the volume and surface forces of the fluid, respectively.

\subsection{Fluid Field Analysis of High-Speed Permanent Magnet Synchronous Traction Motor}

The analysis of the temperature rise in high-speed permanent magnet synchronous motors is inseparable from the ventilation and cooling system of the motors. The high-speed permanent magnet synchronous traction motors are axially forced air-cooled. The heat dissipation is mainly achieved by the cooling air through the internal and external air ducts of the stator [11]. The stator of a permanent magnet motor uses toroidal windings. While reducing the length of the motor's end, it can also increase the cooling area of the stator windings so that the stator windings are directly cooled by the airflow, improving the cooling efficiency [12]. The ventilation cooling system structure of the high-speed permanent magnet synchronous traction motor discussed in this study is shown in Fig. 1.

The flow state of a fluid can be divided into two types, i.e., laminar and turbulent, which are mainly distinguished according to the nature of the fluid, the speed of the flow rate, and the flow path. The Reynolds number is a parameter that determines the flow characteristics of viscous fluids. The rotor surface speed of high-speed permanent magnet motors is very high, and the Reynolds number of the fluid in the air gap is large, which is a turbulent state. Under the conditions of the turbulent flow field, according to the theory of viscous fluid mechanics and the law of physical conservation, the expressions and general forms of general control equations of the flow field inside high-speed permanent magnet motors can be obtained, as shown in Eqs. (4) and (5): 


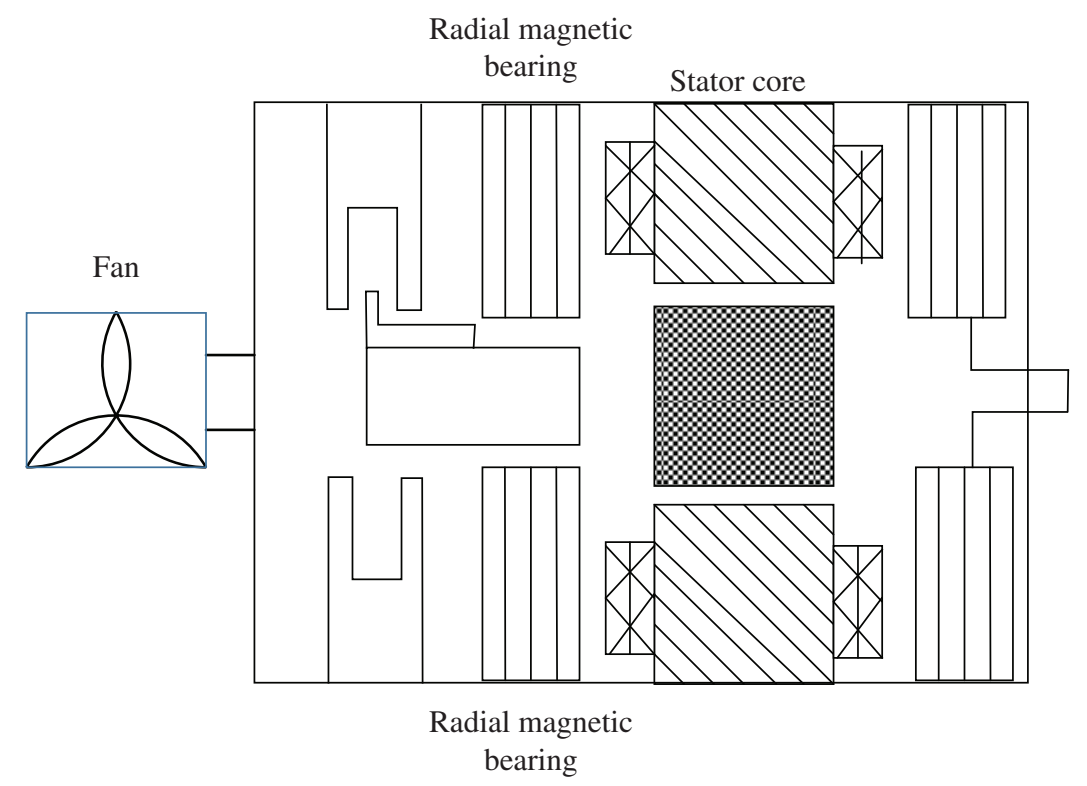

Figure 1: Schematic diagram of the ventilation and cooling system of high-speed permanent magnet synchronous traction motor

$\frac{\partial}{\partial t}(\rho \phi)+\nabla \cdot\left(\rho u_{j} \phi\right)=\nabla \cdot(\mathrm{r} \nabla \varphi)+\mathrm{S}$
$\frac{\partial}{\partial t}(\rho \phi)+\frac{\partial}{\partial x_{j}}\left(\rho u_{j} \phi\right)=\frac{\partial}{\partial x_{j}}\left(\mathrm{r} \frac{\partial \phi}{\partial x_{j}}\right)+S$

The above equations are integrated by using the finite volume method, and the following expression is obtained:

$\frac{\partial}{\partial t} \iint{ }_{\nu} \rho \phi d V+\oiint_{A} \rho \phi u n d A=\oiint_{A} \nabla_{\phi} n d A+\iiint{ }_{v} S_{\phi} d V$

where $\phi$ represents a universal variable, $\mathrm{r}$ is a diffusion coefficient, while $S$ and $S_{\phi}$ are generalized source terms of $\phi$.

The finite element method (FEM) is a modern computing method that has developed rapidly with the development of electronic computers [13]. It is an effective numerical analysis method that was first applied in the field of continuum mechanics-analysis of static and dynamic characteristics of aircraft structures, and was soon widely used to solve continuity problems such as heat conduction, electromagnetic fields, and fluid mechanics. The finite element model can model a solid of any shape, that is, it can simulate the heat conduction phenomenon in a solid of any shape. In the finite element analysis, the concept of equivalent air gap thickness in the thermal circuit method cannot be used to simulate contact thermal resistance. The reason is that the equivalent air gap thickness is very small compared to the motor size parameter, which cannot be modeled. In Ansys, the concept of "contact unit" can be used to simulate the heat conduction phenomenon of the contact surface. In Ansys, the contact heat transfer is defined as: 
$q=T C C \times\left(T_{t}-T_{c}\right)$

where: $q$ is the heat transfer amount per unit area, TCC is the thermal contact conduction coefficient, and $T$ and $T_{c}$ are the temperature of the two contact surfaces.

When using the contact setting of Ansys, it is necessary to set the surface elements of the two contact surfaces as a group of target surface and contact surface elements, and assign the same real constant TCC. Among them, the derivation process of $T C C$ is:

$q=\frac{1}{A} \times \frac{1}{R} \times\left(T_{t}-T_{c}\right)$

where: $A$ is the contact area, $R$ is the contact thermal resistance.

In summary, in addition to the FEM that can accurately model the heat conduction of solid components, the setting of key parameters still depends on empirical data.

The theoretical study of the finite formula method (FFM) mainly focuses on the construction of the symmetric positive definite constitutive matrix and the discrete format of the FFM [14]. For the coupling field calculation problem based on the FEM, due to the highly nonlinear coupling field problem, the corresponding conditional variation principle does not exist, or the corresponding weak form structure is too complicated. In addition, due to the differences in the requirements of each physical field for the mesh and the complicated pre-processing, its calculation scale is too huge and has practical difficulties. The FFM directly derives the field governing equations in the form of algebraic equations based on the laws of physical fields, and its coupling terms are easy to be constructed. It skips the process of the FEM that requires the construction of conditional variational principles or weak forms, and only needs to reconstruct the constitutive matrix.

\subsection{Calculation of Temperature Rise in High-Speed Permanent Magnet Synchronous Traction Motor Based on Equivalent Thermal Circuit Method}

The equivalent thermal circuit method is a common method for calculating the temperature rise in a motor. The similarity between heat transfer and circuit theory is used to reduce the temperature field to a thermal circuit with centralized parameters for the calculation. The distributed real heat source and thermal resistance are replaced with the concentrated heat source and thermal resistance, forming an equivalent thermal circuit [15]. The accurate calculation of the equivalent thermal circuit method mainly depends on parameters such as thermal resistance and heat dissipation coefficient, which needs to be obtained by solving the velocity field of the motor fluid.

Before analyzing the thermal circuit, it is necessary to consider whether there is heat exchange between the stator and the rotor. Due to the large axial airflow of the high-speed permanent magnet synchronous traction motor, the heat exchange between the stator and the rotor can be ignored. Therefore, the stator and rotor of the motor can form their thermal paths [16]. The core and windings of the stator are the heat source of the stator thermal circuit, while the permanent magnet and the sheath are the heat source of the rotor thermal circuit. Fig. 2 is an axial sectional view of a high-speed permanent magnet motor.

The calculation of the temperature rise in high-speed motors is mainly the temperature rise in the specified sub-core and windings, as well as the temperature rise in the rotor sheath and permanent magnets. Meanwhile, the temperature rises in these components change in space according to a certain law. Although the heating of the motor is based on the highest temperature rise, due to the certain relationship between the highest temperature rise and the average temperature rise, the average temperature rise in the heating components can be calculated to evaluate the heat generation of the motor. The heat of high-speed motors is transmitted to the cooling air from the rotor and stator windings through 
conductive and thermal resistances. The conductive and thermal resistances inside the motor can be expressed as Eqs. (13) and (14), respectively:

$R_{\lambda}=\frac{\delta}{\lambda S}$

$R_{\alpha}=\frac{1}{\alpha S}$

where $\lambda$ indicates the thermal conductivity of the material, $\delta$ refers to the length of the heat path, $\alpha$ refers to the heat dissipation coefficient, and $S$ refers to the heat dissipation area.

The concentrated parameter equivalent thermal circuit diagrams of the high-speed permanent magnet motor stator and rotor are shown in Figs. 3 and 4, respectively.

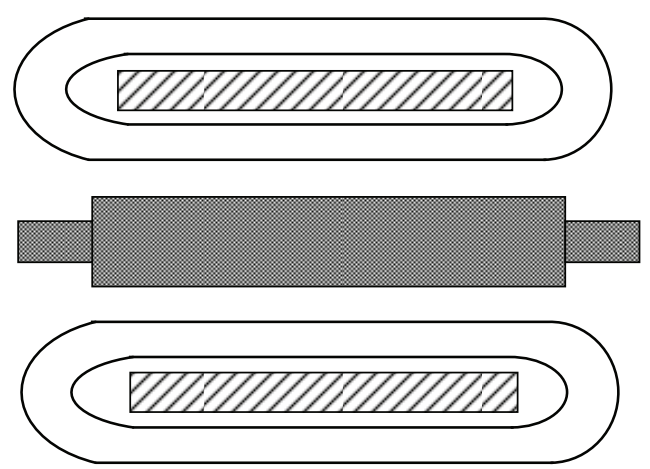

Figure 2: The axial sectional view of the high-speed permanent magnet motor

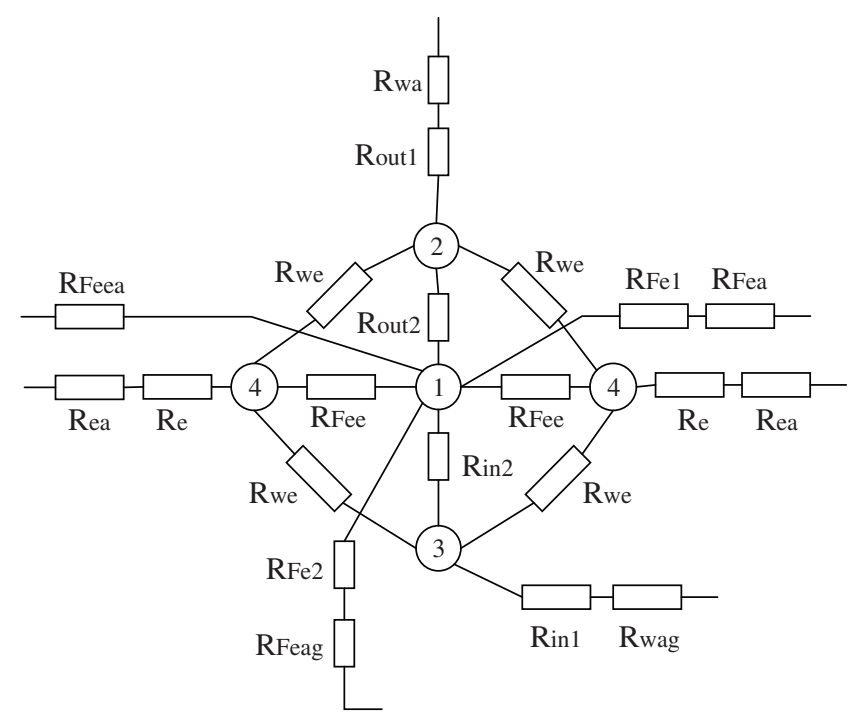

Figure 3: Equivalent thermal circuit diagram of concentrated parameters of high-speed permanent magnet motor stator 


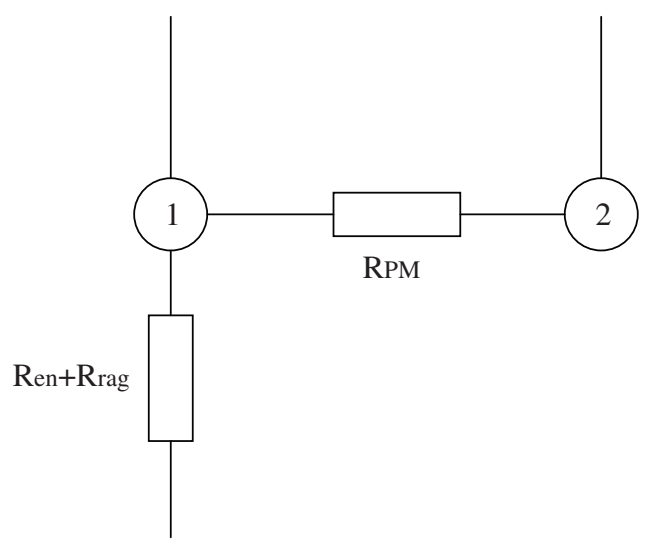

Figure 4: Equivalent thermal circuit diagram of concentrated parameters of high-speed permanent magnet motor rotor

The heat dissipation thermal resistance in the equivalent thermal resistance refers to the thermal resistance between the core and the winding and the ambient air and is jointly determined by the heat dissipation coefficient $\alpha$ and the heat dissipation area $S$. It can be divided into the thermal resistance RFea of the stator core's external ventilation channel environment, the axial thermal resistance Rwa of the thermal resistance of the winding's external ventilation environment, and the environmental thermal resistance Rea of the winding end.

\subsection{Thermal Circuit Model of High-speed Permanent Magnet Synchronous Traction Motor}

The composition of high-speed permanent magnet synchronous traction motor includes end cover, mounting plate, casing, stator core (yoke, the tooth), stator winding (slot winding, end winding), rotor core, rotating shaft, bearing, air gap, and air inside the machine. The motor is an axisymmetric structure; therefore, all the temperature changes in the circumferential direction are ignored except the temperature difference between the windings in the slot and the teeth of the stator core [17]. The motor is divided into a plurality of hollow cylindrical units with a rotating shaft as the center, and the conductive thermal resistance of each cylindrical component can be calculated by the equation. The size of each tooth of the motor is the same. Therefore, the motor can be modeled by the equivalent thermal resistance of the iron cores connected in parallel. Similarly, this method is also applicable to the stator windings of the motor. Since most permanent magnet synchronous motors use the B35 end-face mounting type, the mounting surface significantly increases the heat dissipation area of the motor and has a greater impact on the temperature rise in the motor [18]. The convective heat conduction in the motor mainly occurs between the outside air and the motor's casing, end cover, and mounting plate, and between the air inside the machine and the stator core, rotor core and end cover. The thermal circuit model includes these two types of convection heat resistance.

\subsection{Common Cooling Methods for High-speed Permanent Magnet Synchronous Traction Motors}

To ensure the effective operation of the motor, the motor windings and cores must be cooled, and the temperature of each part must be controlled.

Within the allowable range, the current common cooling methods include air cooling, evaporative cooling, water cooling, and oil cooling.

(1) Air cooling uses flowing air as a cooling medium, and the flowing air takes away the heat generated by the motor itself. The air-cooling system has a simple structure and is widely used. It can be used from micro-hydrogen generators to giant hydro-generators [19]. However, the disadvantage of air 
cooling is that the low thermal conductivity of the air will cause the temperature of the stator windings and the core to rise too high, affecting the insulation life. In addition, with the increase of the motor capacity, more heat will be generated at the same time as the electromagnetic loss increases. Intelligently reducing the temperature rise by increasing the wind speed or increasing the air volume will increase the wind wear of the motor.

(2) Water cooling is a cooling method based on air cooling and meets more motor heat dissipation requirements. It is often used in turbo-generators. However, its reliability is lower than air cooling. The water cooling causes the heat generated by the stator winding losses in the generator to no longer pass through the core, and the temperature rise in the core will be greatly reduced [20]. However, in the application process of water-cooling technology, a pure water treatment system needs to be set. If the system does not purify the water sufficiently, it will cause water blocking and affect the cooling effect.

(3) The commonly used oil cooling medium is hydraulic engine oil. Since the cooling channels of the inner slots of the stator are oil-cooled, it is necessary to isolate the internal cooling channels from the air gap and the rotor.

(4) In the evaporative cooling technology, the stator windings of generators combine hollow copper wires and solid copper wires with each other and use the latent heat of vaporization during fluid boiling for heat dissipation [21]. Since the latent heat of vaporization of the fluid is much larger than the specific heat of the fluid, the cooling effect of evaporative cooling is more significant. When the motor adopts the evaporative cooling method, the cooling effect of the evaporative cooling is significant so that the temperature rise in the entire motor is reduced, the temperature distribution of the motor is uniform, and the operation stability is improved.

\subsection{Cooling Effect of High-Speed Permanent Magnet Synchronous Traction Motor}

When a high-speed permanent magnet motor is running, a variety of losses will occur inside; these losses will be converted into heat and spread to the surroundings, which will eventually increase the temperature of each part of the motor. If the temperature inside the motor exceeds the temperature allowed by the insulation, it will cause the motor to lose magnetism or even be damaged, which directly affects the rated power and service life of the motor. Therefore, it is particularly important to solve the problem of motor temperature rise. It is necessary to start from reducing losses and heat generation, and at the same time, to improve the cooling and heat dissipation capacity of the motor. This study investigates the cooling method of the same 24-slot high-speed permanent magnet motor used in the previous experiments. Based on the theory of fluid mechanics, a three-dimensional fluid field model of oil cooling, air cooling, water cooling, and mixed cooling is established. Boundary conditions and fluid-solid coupled temperature field calculations verify the effectiveness of the analysis method and the feasibility of the cooling structure.

When the high-speed permanent magnet motor is running at 15,000 r/min at no load, the internal cooling channel of the stator inner slot uses oil cooling, and the temperature of the stator winding is basically the same as the temperature of the cooling medium. Therefore, the temperature of the motor will increase as the temperature of the cooling oil increases. In this study, the high-speed permanent magnet synchronous traction motor uses an oil suspension bearing to support the motor system. The oil tile and the motor cooling oil share a cooling system. When the motor speed increases, the losses caused by the oil tiles will increase rapidly, which will cause the cooling oil temperature of the motor to rise rapidly. When the oil temperature of the cooling system exceeds $50^{\circ} \mathrm{C}$, the oil pressure will drop too fast, which will reduce the efficiency of the oil circulation system. This requires the oil supply pressure to be kept constant, and the working pressure control device of the lubrication system to have high accuracy, so as to avoid causing insufficient lubrication of the local lubrication system and the wear and sintering of oil film bearings. 


\section{Results and Discussion}

\subsection{Calculation Results and Analysis of Temperature Field in High-Speed Permanent Magnet Motor}

The temperature field in the motor can be calculated only after the losses in each part of the motor are accurately calculated. In the solution domain, the unit where each part of the motor is located can be used as a heat source for loss. The loss of high-speed permanent magnet synchronous traction motor mainly includes iron loss, mechanical loss, copper loss of stator winding, rotor eddy current loss, and other losses. In this experiment, by analyzing the losses of the 24-slot high-speed permanent magnet synchronous traction motor, the losses of each part of the motor can be calculated. The specific results are shown in Tab. 1.

Table 1: Loss of each component of high-speed induction motor $(\mathrm{kW})$

\begin{tabular}{ll}
\hline Loss of each component & Data \\
\hline Stator core loss & 6.16 \\
Copper consumption of stator winding & 6.58 \\
Eddy current loss of rotor & 39.55 \\
Mechanical loss & 12.98 \\
Additional loss & 7.54 \\
\hline
\end{tabular}

In this study, by analyzing the fluid-solid coupled temperature field of the motor, it can be obtained that the high-speed permanent magnet synchronous traction motor has a stator temperature of $68^{\circ} \mathrm{C}$ and $76^{\circ} \mathrm{C}$ at the no-load operation of $50,000 \mathrm{r} / \mathrm{min}$ and $60,000 \mathrm{r} / \mathrm{min}$, respectively. The temperature of the air outlet side of the inner air duct is the highest, which is $100^{\circ} \mathrm{C}$ at the rated speed. Fig. 5 is the influence curve of the stator axial wind speed change of the high-speed permanent magnet synchronous traction motor on the stator winding temperature, as well as the influence curve of the motor axial wind speed on the rotor temperature rise. It can be seen from the figure that the temperature rise in the stator winding and the rotor decreases with the increase of the axial ventilation speed.

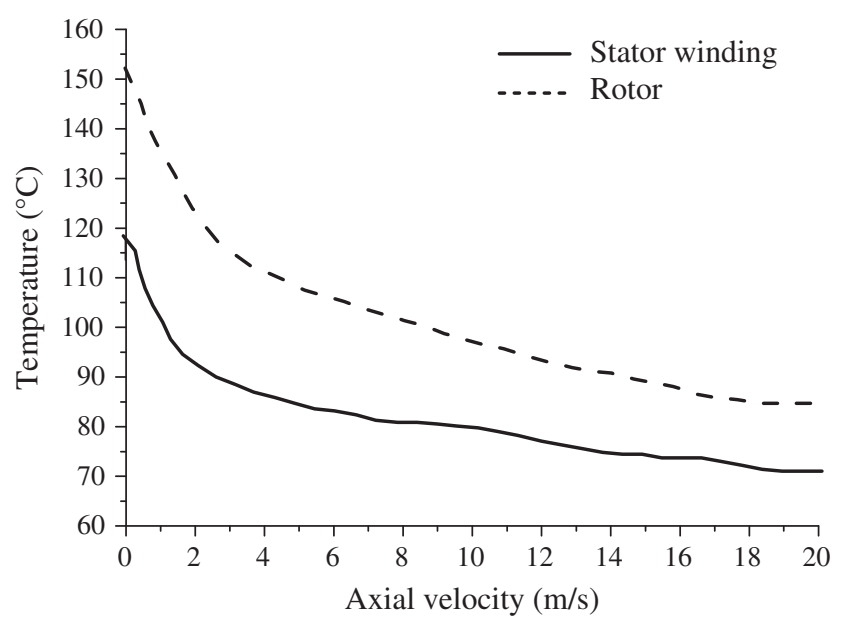

Figure 5: The influence of the axial wind speed of the motor on the temperature rise in the stator and rotor

To verify the correctness of the motor temperature rise calculation by thermal circuit method and fluidsolid coupling analysis, a temperature rise test is performed on a 24-slot high-speed permanent magnet motor prototype. The thermistor is used to measure the respective temperature rise in the stator winding and core of 
the motor and place them in the inner and outer tanks, respectively. By monitoring the temperature of the air outlets inside and outside the motor at different speeds, it is found that the motor reached a stable temperature rise after $65 \mathrm{~min}$ of operation. The temperature change curves of the internal and external vents and the stator when the motor is at 50,000 r/min and 60,000 r/min are shown in Figs. 6 and 7.

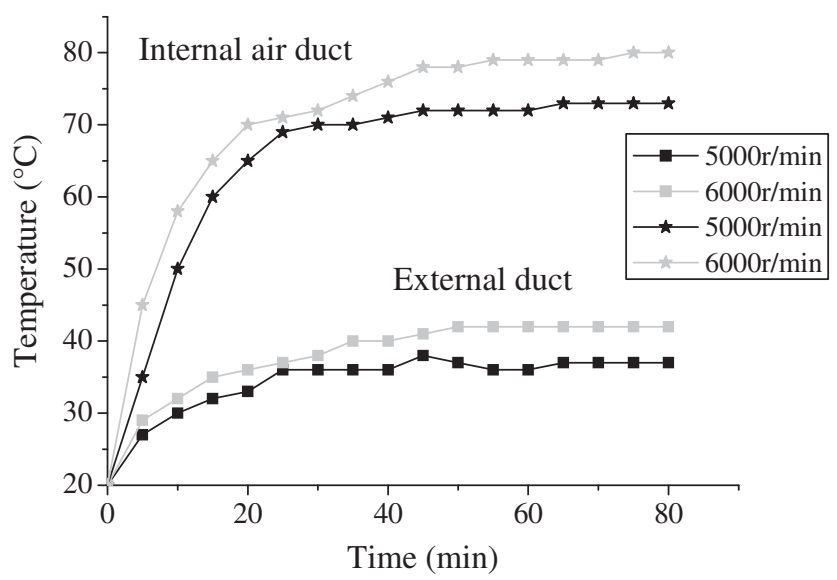

Figure 6: Wind temperature change curve of the air duct inside and outside the motor

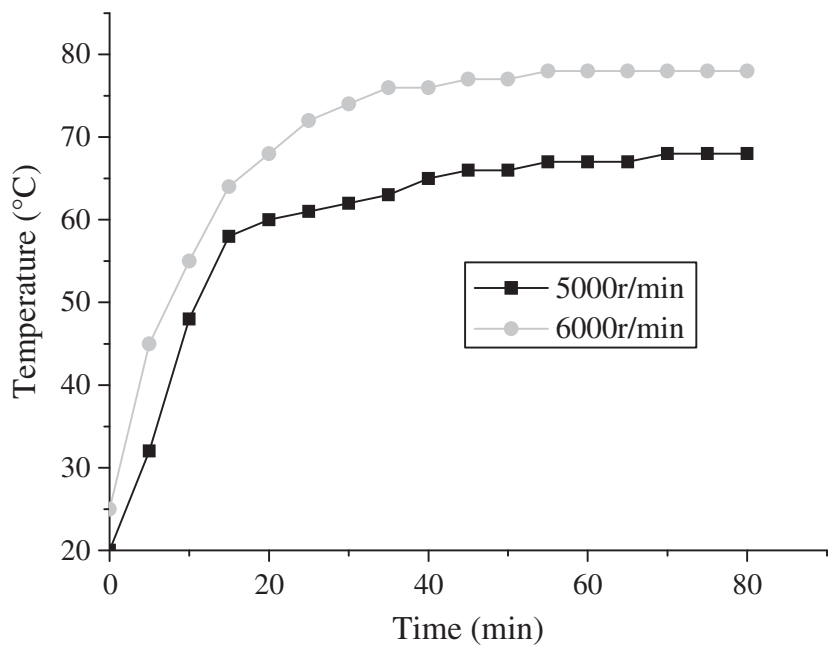

Figure 7: Motor stator temperature change curve at different speeds

\subsection{Three-Dimensional Temperature Field Analysis of Permanent Magnet Motor}

In the process of finite element analysis, for many practical engineering problems, some regions of the model will produce large strain, which will cause the element distortion, so that the solution cannot be carried out or the solution result is incorrect. Therefore, the mesh must be automatically re-divided. Under certain conditions, the smaller the mesh is, the more the nodes are, and the higher the accuracy is. Under the same grid, solution degrees of freedom, and iterative algorithms, when the memory occupation is the same, the FFM calculation time and iteration times are less than those of the FEM, and the convergence speed of the former is faster. In terms of time, the calculation time of the FFM is $50 \%, 85.5 \%, 84.8 \%$, and $86.5 \%$ of the FEM. Fig. 8 shows the corresponding relation curve. The reason is that the coefficient matrix obtained by the FFM dispersion under the same grid has a smaller spectral radius and condition 
number than the FEM. Its calculation stability and convergence are superior to the FEM. Besides, with the refinement of the grid, the spectral radius of the coefficient matrix obtained by the two dispersions shows a decreasing trend. FFM1 FFM5 are the calculated values of the FFM when the grid number is 150, 522, 3032, 18934, and 419034, as shown in Fig. 9.

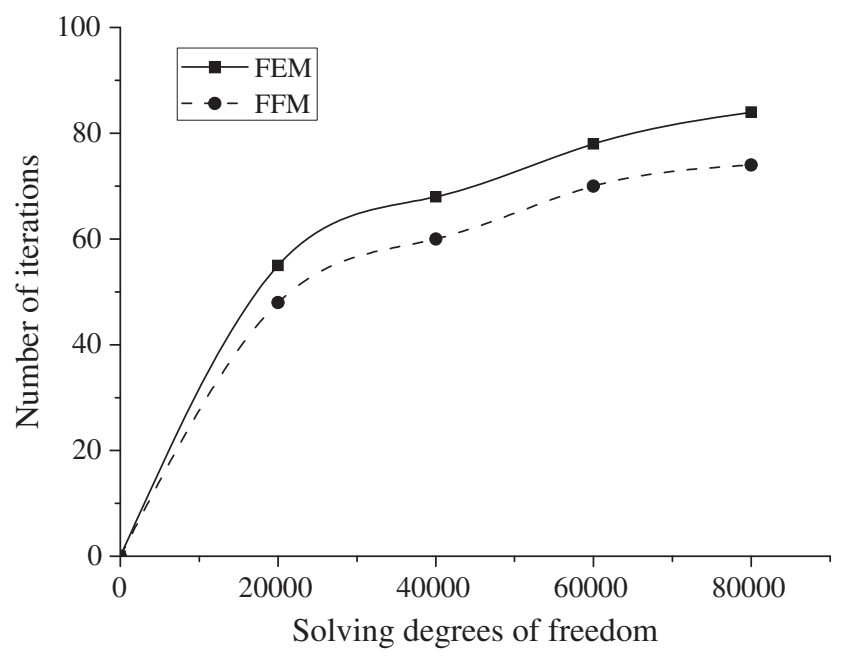

Figure 8: Relations among FFM, FEM calculation iterations, and solution degrees of freedom

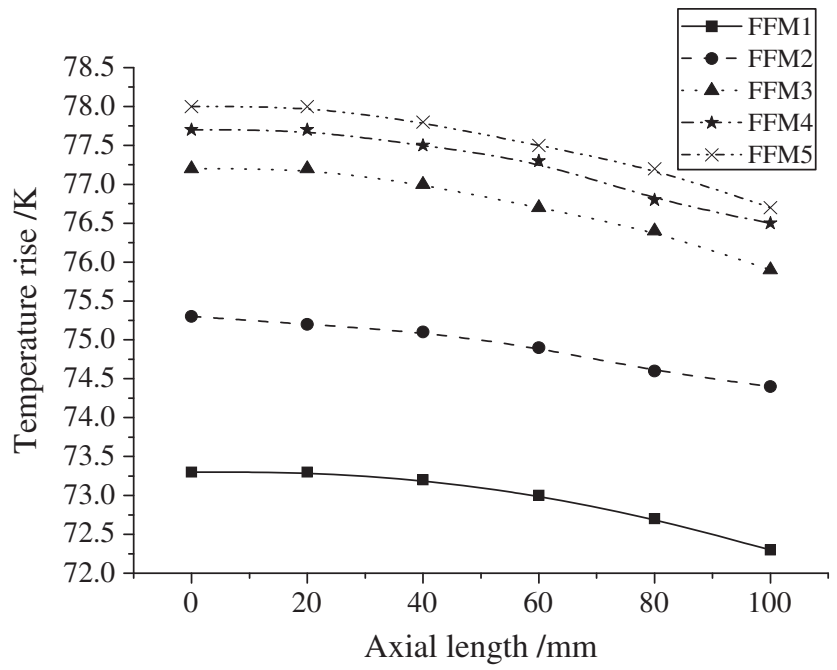

Figure 9: Calculated values of FFM under different grid numbers

\subsection{Research Results of Cooling Methods for High-Speed Permanent Magnet Motors}

This study first uses separate air cooling for a 24-slot motor. It is found that the temperature rise in the permanent magnet at the rated speed has exceeded the limit temperature requirement of the permanent magnet, which is prone to overheating and demagnetization. Therefore, this study proposes an improved water-air cooling method. Considering the requirements of the rotor cooling of the sample motor, as well as the characteristics of air cooling and water cooling, a mixed cooling method of ventilation cooling in the inner air duct and water cooling of the rotor and the stator casing is proposed. When keeping the wind speed of the inner air duct constant for no-load operation, the cooling water volume corresponding 
to the water speed of the cooling channel of the casing is shown in Tab. 2. When the water velocity of the cooling channel of the casing is $0.05 \mathrm{~m} / \mathrm{s}$, the cooling water volume is $6 \times 10^{-5} \mathrm{~m}^{3} / \mathrm{s}$. When the water velocity of the cooling channel of the casing is $0.1 \mathrm{~m} / \mathrm{s}$, the cooling water volume is $1.3 \times 10^{-4} \mathrm{~m}^{3} / \mathrm{s}$. When the water velocity of the cooling channel of the casing is $0.2 \mathrm{~m} / \mathrm{s}$, the cooling water volume is $2.7 \times 10^{-4} \mathrm{~m}^{3} / \mathrm{s}$. When the water velocity of the cooling channel of the casing is $0.4 \mathrm{~m} / \mathrm{s}$, the cooling water volume is $5.3 \times 10^{-4} \mathrm{~m}^{3} / \mathrm{s}$. When the water velocity of the cooling channel of the casing is $0.8 \mathrm{~m} / \mathrm{s}$, the cooling water volume is $1.1 \times$ $10^{-3} \mathrm{~m}^{3} / \mathrm{s}$. When the water velocity of the cooling channel of the casing is $1.0 \mathrm{~m} / \mathrm{s}$, the cooling water volume is $1.34 \times 10^{-3} \mathrm{~m}^{3} / \mathrm{s}$. By analyzing the temperature rise in a 24-slot mixed-cooling high-speed permanent magnet motor under no-load conditions, it can be known that the introduction of water cooling and air cooling at the casing can reduce the temperature rise in the motor rotor. The hybrid cooling structure of axial air cooling for the inner air duct of the motor and water cooling for the stator casing used in this study can meet the design requirements of high-speed permanent magnet motors for ventilation and cooling of permanent magnets.

Table 2: Volume of cooling water of 24-slot permanent magnet motor at different water speeds

\begin{tabular}{ll}
\hline Cooling channel water speed $(\mathrm{m} / \mathrm{s})$ & Cooling water volume $\left(\mathrm{m}^{3} / \mathrm{s}\right)$ \\
\hline 0.05 & $6 \times 10^{-5}$ \\
0.1 & $1.3 \times 10^{-4}$ \\
0.2 & $2.7 \times 10^{-4}$ \\
0.4 & $5.3 \times 10^{-4}$ \\
0.8 & $1.1 \times 10^{-3}$ \\
1.0 & $1.34 \times 10^{-3}$ \\
\hline
\end{tabular}

\section{Discussion}

Due to its high-speed and high winding current frequency, the high-speed permanent magnet synchronous traction motor has a significantly higher loss than a normal motor of the same power level. Considering that the problem of motor temperature rise is related to the increase of power density and the reduction of overall heat dissipation area, in the design of high-speed permanent magnet synchronous traction motors, the effective heat dissipation and cooling methods are essential. First, the theoretical basis of heat transfer and fluid mechanics is discussed, the fluid field of a high-speed permanent magnet synchronous traction motor is analyzed, and the equivalent thermal circuit method is introduced into the calculation of the temperature rise of the motor. Finally, the problems of stator, rotor loss, motor temperature rise, and cooling method of the high-speed permanent magnet synchronous motor are explored through experiments.

In this study, by analyzing the fluid-solid coupling temperature field of a 24-slot high speed permanent magnet synchronous traction motor, it can be obtained that the stator temperature of the high-speed permanent magnet synchronous traction motor at 50,000 r/min and 60,000 r/min at no-load operation is $68^{\circ} \mathrm{C}$ and $76^{\circ} \mathrm{C}$, respectively. The thermal circuit coupling computational fluid dynamics is a method that can calculate the average temperature rise of each component and can also comprehensively calculate the temperature of each local point. Then, this study conducts a temperature rise test on the prototype of the motor. By monitoring the temperature of the air outlets inside and outside the motor at different speeds, it is found that the motor reached a stable temperature rise after $65 \mathrm{~min}$ of operation. 


\section{Conclusions}

To solve the problem of temperature rise of the motor, this study explores the cooling method of the motor. The temperature rise of the 24-slot mixed-cooled high-speed permanent magnet motor is analyzed under no-load conditions. The results show that the introduction of water cooling and air cooling at the casing can reduce the motor rotor temperature rise. In this study, the hybrid cooling structure of axial air cooling of the motor inner air duct and water cooling of the stator casing can meet the design requirements of high-speed permanent magnet motors for ventilation and cooling of permanent magnets. However, this study only introduces the no-load experiments of high-speed motors. Most of the motors are in the load state during the operation. Therefore, the motor towing process needs to be investigated to verify the temperature rise of the motors under loads.

Funding Statement: The author(s) received no specific funding for this study.

Conflicts of Interest: The authors declare that they have no conflicts of interest to report regarding the present study.

\section{References}

1. Tong, W., Cheng, X., Sun, J., Chen, P., Tang, R. (2017). Suppression effect of rotor wafters on permanent magnet temperature rise for high-speed permanent magnet motor. Proceedings of the CSEE, 37(5), 1526-1534.

2. Ji-Kai, S. I., Song, H. E., Feng, H. C., Zhang, X. L., Feng, X. H. (2015). Characteristic analysis of surface-mounted and interior hybrid permanent magnet synchronous motor based on equivalent magnetic circuit method. Journal of China Coal Society, 40(5), 1199-1205.

3. Nie, L. N., Zhang, Y., Xiong, W. W., Lim, T. T., Xu, R. et al. (2016). A surfactant-thermal method to prepare crystalline thioantimonate for high-performance lithium-ion batteries. Cheminform, 47(13), 111-116.

4. Marków, M., Janecki, D., Orecka, B., Misiołek, M., Warmuziński, K. (2017). Computational fluid dynamics in the assessment of patients' postoperative status after glottis-widening surgery. Advances in Clinical and Experimental Medicine, 26(6), 947-952. DOI 10.17219/acem/64235.

5. Kumar, M., Memon, Z. A., Uqaili, M. A., Hussain, M. (2018). An experimental insulation testing investigation of 210 MW generator: a case of thermal power station Jamshoro. International Journal of Energy Optimization and Engineering, (6), 68-91. DOI 10.4018/IJEOE.2018100104.

6. Aghazadeh, H., Afjei, E., Siadatan, A. (2019). Comprehensive design procedure and manufacturing of permanent magnet assisted synchronous reluctance motor. International Journal of Engineering, 9(32), 1299-1305.

7. Wang, Z. W., Han, Q. F., Nash, D. H., Liu, P. Q. (2017). Investigation on inconsistency of theoretical solution of thermal buckling critical temperature rise for cylindrical shell. Thin-Walled Structures, 119(8), 438-446. DOI 10.1016/j.tws.2017.07.002.

8. Wei, Z. A., Sonntag, S. J., Toma, M., Singh-Gryzbon, S., Sun, W. (2018). Computational fluid dynamics assessment associated with transcatheter heart valve prostheses: A position paper of the ISO working group. Cardiovascular Engineering \& Technology, 9(19), 1-11.

9. Asef, P., Bargalló Perpiñá, R. (2016). Losses calculation of an aerospace retraction wheel motor with regarding to electromagnetic-field analysis investigation. Journal of Energy and Power Engineering, 100(10), 183-190.

10. Jha, B. K., Gambo, Y. Y. (2020). Transient natural convention heat and mass transfer flow in a vertical channel in the presence of Soret and Dufour effects: An analytical approach. International Journal of Modern Physics C, 31(2), 455.

11. Bencherif, M., Brahmi, B. N. (2018). New method of parameters extraction of the solar panels. Journal of Sustainable Energy Engineering, 1(6), 11-47. DOI 10.7569/JSEE.2017.629517.

12. Mirrashid, N., Rakhtala, S. M., Ghanbari, M. (2018). Robust control design for air breathing proton exchange membrane fuel cell system via variable gain second-order sliding mode. Energy Science \& Engineering, 3(6), 126-143. DOI 10.1002/ese3.199.

13. Zeng, W., Liu, G. R. (2018). Smoothed finite element methods (S-FEM): An overview and recent developments. Archives of Computational Methods in Engineering, 25(2), 397-435. DOI 10.1007/s11831-016-9202-3. 
14. Tomeo, R., Pitilakis, D., Bilotta, A. (2018). SSI effects on seismic demand of reinforced concrete moment resisting frames. Engineering Structures, 173, 559-572. DOI 10.1016/j.engstruct.2018.06.104.

15. Cao, R., Zhang, Z., Jin, Y., Zhang, L. (2017). Double-sided linear flux-switching permanent magnet motor with yokeless secondary and control system. Proceedings of the CSEE, 37(22), 6585-6593.

16. Yong, L., Li, B., Xing, X., Sun, X. (2017). A nonlinear decoupling control approach using RBFNNI-based robust pole placement for a permanent magnet in-wheel motor. IEEE Access, 20(6), 1844-1854.

17. Hossain, M. F. (2016). Theoretical modeling for hybrid renewable energy: An initiative to meet the global energy. Journal of Sustainable Energy Engineering, 1(4), 5-36. DOI 10.7569/JSEE.2016.629505.

18. Moubarak, A., El-Saady, G., Ibrahim, E. N. A. (2017). Variable speed photovoltaic water pumping using affinity laws. Journal of Power and Energy Engineering, 5(11), 50-71. DOI 10.4236/jpee.2017.511005.

19. Samami, M., Niaz Azari, M., Pahnekollaei, A. (2017). Optimal design of a brushless DC motor, by cuckoo optimization algorithm (research note). International Journal of Engineering, 5(30), 668-677.

20. Tsuji, T., Ikado, K., Koizumi, H., Uchiyama, S., Kajimoto, K. (2017). Difference in intracellular temperature rise between matured and precursor brown adipocytes in response to uncoupler and $\beta$-adrenergic agonist stimuli. Scientific Reports, 7(1), 12889. DOI 10.1038/s41598-017-12634-7.

21. Zhang, P., Song, C., Wu, Z., Lü, G. (2018). Influence of last-post-injection strategy on exhaust emissions and temperature rise behaviour of a DOC during DPF active regeneration. Chinese Internal Combustion Engine Engineering, 39(3), 45-52. 